\title{
COMPLETE SOLUTIONS TO FAMILIES OF QUARTIC THUE EQUATIONS
}

\author{
ATTILA PETHÖ
}

\begin{abstract}
Using a method due to E. Thomas, we prove that if $|a|>9.9 \cdot 10^{27}$ then the Diophantine equations

$$
x^{4}-a x^{3} y-x^{2} y^{2}+a x y^{3}+y^{4}=1
$$

and

$$
x^{4}-a x^{3} y-3 x^{2} y^{2}+a x y^{3}+y^{4}= \pm 1
$$

have exactly twelve solutions, namely $(x, y)=(0, \pm 1),( \pm 1,0),( \pm 1, \pm 1)$, $(\mp 1, \pm 1),( \pm a, \pm 1),( \pm 1, \mp a)$ and eight solutions, $(x, y)=(0, \pm 1)$, $( \pm 1,0),( \pm 1, \pm 1),( \pm 1, \mp 1)$, respectively.
\end{abstract}

\section{INTRODUCTION}

Let $F(x, y) \in \mathbf{Z}[x, y]$ be homogeneous of degree $n \geq 3$ and irreducible. A classical problem of number theory is to solve completely the Diophantine equation

$$
F(x, y)= \pm 1,
$$

commonly known as a Thue equation.

Let $\mathbf{K}=\mathbf{Q}(\partial)$, where $\partial$ denotes one of the roots of $F(x, 1)$. If $n=4$, then the maximal order of $\mathbf{K}$ has unit rank 1, 2, or 3 according as $F(x, 1)$ has four, two, or no imaginary roots. This will be called the unit rank of the Thue equation.

Ljunggren [4] as well as Nagell [5,6] examined the number of solutions of certain classes of quartic Thue equations of unit rank two and one. They proved that the number of solutions is at most 10 and 8 , respectively. Stroeker [10] gave a method which enabled him to solve quartic Thue equations of unit rank two. Using numerical Diophantine approximation techniques, Pethő and Schulenberg [7], Steiner [9], and de Weger [14] solved some single equations of unit rank 3. Stroeker and Tzanakis [11] applied Skolem's $p$-adic method for the same purpose.

Received February 22, 1990; revised September 14, 1990.

1980 Mathematics Subject Classification (1985 Revision). Primary 11D25.

Key words and phrases. Thue equation, linear forms in the logarithms of algebraic numbers.

Research supported by the Hungarian National Foundation for Scientific Research, Grant No. $273 / 86$. 
Recently, all the solutions to infinite parametrized families of cubic Thue equations were found by Thomas [12,13]. His method is based on an A. Bakertype lower bound for linear forms in the logarithms of algebraic numbers.

In this paper we shall use Thomas' method for two classes of quartic Thue equations of unit rank 3. More precisely, let $a$ be an integer and

$$
f_{a}(x, y)=x^{4}-a x^{3} y-x^{2} y^{2}+a x y^{3}+y^{4}=(x-y) x(x+y)(x-a y)+y^{4}
$$

as well as

$$
g_{a}(x, y)=x^{4}-a x^{3} y-3 x^{2} y^{2}+a x y^{3}+y^{4} .
$$

In $\S 2$ we show that if $|a| \neq 2$, then $f_{a}(x, y)$ is irreducible, and similarly, if $a \neq 0$, then $g_{a}(x, y)$ is irreducible.

It is easy to check that

$$
f_{a}(x, y)=1
$$

is solved by $(x, y)=(0, \pm 1),( \pm 1,0),( \pm 1, \pm 1),(\mp 1, \pm 1),( \pm a, \pm 1)$, $( \pm 1, \mp a)$, which we call trivial solutions. The only nontrivial solutions we know correspond to $|a|=4$, namely $(x, y)=( \pm 8, \pm 7),( \pm 7, \mp 8)$ for $a=4$, and $( \pm 8, \mp 7),( \pm 7, \pm 8)$ for $a=-4$.

Our main result is

Theorem 1. The equation

$$
f_{a}(x, y)=m, \quad m= \pm 1,
$$

is solvable only for $m=1$. If $|a|>9.9 \cdot 10^{27}$, then (1.2) has only the trivial solutions.

Using the reduction procedure of Baker and Davenport [1], more precisely its implementation by Gaál and Schulte [3], we were able to prove

Theorem 2. If $3 \leq|a| \leq 100$, then (1.2) has only the trivial solutions except for $|a|=4$, when it has the four nontrivial solutions given above.

Similarly,

$$
g_{a}(x, y)= \pm 1
$$

has the trivial solutions $(x, y)=(0, \pm 1),( \pm 1,0),( \pm 1, \pm 1),( \pm 1, \mp 1)$. For $|a|=1$ we found four nontrivial solutions, namely $(x, y)=( \pm 2, \pm 1)$, $( \pm 1, \mp 2)$ for $a=1$ as well as $(x, y)=( \pm 2, \pm 1),( \pm 1, \pm 2)$ for $a=-1$. Using the method of the proofs of Theorem 1 and Theorem 2, we get

Theorem 3. If $0<|a| \leq 100$ or $|a|>9.9 \cdot 10^{27}$, then (1.3) has only trivial solutions except for $|a|=1$, when it has the four nontrivial solutions given above.

Comparing these theorems with the above-mentioned results of Ljunggren and Nagell, we see that quartic Thue equations of unit rank three may have more solutions than those of smaller unit rank. 
Let $\eta>1$ be a real quadratic unit with conjugate $\eta^{\prime}$, and let

$$
R_{n}=\frac{\eta^{n}-\eta^{\prime n}}{\eta-\eta^{\prime}}
$$

for $n \in \mathbf{Z}$. It is easy to see that $R_{n} \in \mathbf{Z}$ for all $n \in \mathbf{Z}$. Using the results of Theorems 1, 2, and 3, we also prove

Theorem 4. Assume that $0<\left|\eta+\eta^{\prime}\right| \leq 100$ or $\left|\eta+\eta^{\prime}\right|>9.9 \cdot 10^{27}$ and

$$
4 u^{2}+v^{2}=z^{2}
$$

with $(u, v)=\left(R_{n}, R_{n+1}\right)$ or $\left(R_{n+1}, R_{n}\right)$ and $z \in \mathbf{Z}$. Then $n=0$, or $n=$ 2,-3 (where $\eta \eta^{\prime}=1$ ), except when either $\left|\eta+\eta^{\prime}\right|=4, \eta \eta^{\prime}=1, n=4$, $(u, v)=(56,15)$, and $n=-5,(u, v)=(-56,-15)$ or $\left|\eta+\eta^{\prime}\right|=1, \eta \eta^{\prime}=$ $-1, n=3,(u, v)=(2,3)$, and $n=-4,(u, v)=(-2,-3)$.

\section{ELEMENTARY PROPERTIES OF THE POLYNOMIALS}

Let $a, b \in \mathbf{Z}$ and $f_{a, b}(x)=x^{4}-a x^{3}+b x^{2}+a x+1$. Then we have

Lemma 2.1. Let $\varphi$ be a zero of $f_{a, b}(x)$ and $\sigma: Q(\varphi) \rightarrow Q(\varphi)$ a mapping with $\sigma(\varphi)=-1 / \varphi$. Then $\sigma$ is an automorphism of the field $Q(\varphi)$ and of the module $\mathbf{Z}[\varphi]$. Moreover, the maximal invariant subfield of $Q(\varphi)$ corresponding to $\sigma$ is $Q(\varphi-1 / \varphi)$.

Proof. The first assertion is true because $-1 / \varphi$ is also a root of $f_{a, b}(x)$.

We have

$$
-1 / \varphi=\varphi^{3}-a \varphi^{2}+b \varphi+a \in \mathbf{Z}[\varphi],
$$

which proves the second assertion.

Let $\mathbf{L}$ denote the maximal invariant subfield of $\mathbf{Q}(\varphi)$ corresponding to $\sigma$. Then the degree of $\mathbf{Q}(\varphi)$ over $\mathbf{L}$ is equal to the order of $\sigma$, which is either one or two.

Let $\eta=\varphi-1 / \varphi$. Then $\mathbf{Q}(\eta)$ is a subfield of $\mathbf{L}$ because $\sigma(\eta)=\eta$. Thus, if $\varphi \notin \mathbf{Q}(\eta)$, then $[\mathbf{Q}(\varphi): \mathbf{Q}(\eta)]=2$ and $\mathbf{L}=\mathbf{Q}(\eta)$.

We have

$$
f_{a, b}(x)=x^{2}\left(\left(x-\frac{1}{x}\right)^{2}-a\left(x-\frac{1}{x}\right)+b+2\right),
$$

hence $\eta$ is a zero of the polynomial $y^{2}-a y+(b+2)$, i.e., $\eta$ is of degree one or two.

Assume that $\varphi \in \mathbf{Q}(\eta)$. If $\eta \in \mathbf{Q}$, then $\mathbf{Q}(\varphi)=\mathbf{Q}=\mathbf{L}$, otherwise $[\mathbf{Q}(\eta)$ : $\mathbf{Q}]=2$, and there exist $u, v \in \mathbf{Q}$ with $\varphi=u+v \eta$. Then $\sigma(\varphi)=u+v \sigma(\eta)$, and by $\sigma(\eta)=\eta$ we have $-1 / \varphi=u+v \eta=\varphi$ or $\varphi^{2}=-1$. Also in this case the mapping $\sigma$ is of order one, i.e., $\mathbf{L}=\mathbf{Q}(\eta)$. The lemma is proved.

It is clear that $f_{a}(x, 1)=f_{a,-1}(x)$ and $g_{a}(x, 1)=g_{a,-3}(x)$. 
Corollary 2.2. Let $a \in \mathbf{Z}$. If $|a| \neq 2$, then $f_{a}(x, 1)$ is irreducible, and similarly, if $a \neq 0$, then $g_{a}(x, 1)$ is irreducible.

Proof. Let $\varphi$ be a root of $f_{a}(x, 1)$, and $\eta=\varphi-1 / \varphi$. Then $\eta$ is a zero of $y^{2}-a y+1$. It has degree two over $\mathbf{Q}$ except when $|a|=2$. From the proof of Lemma 2.1 it follows that $[\mathbf{Q}(\varphi): \mathbf{Q}(\eta)]=2$, hence $[\mathbf{Q}(\varphi): \mathbf{Q}]=4$. The proof of the other assertion is similar.

Let $a \geq 0$ and $\alpha$ be the largest real root of $f_{a}(x, 1)=0$, and set $\varepsilon=\alpha-1 / \alpha$. Then $\varepsilon$ is a zero of the polynomial $y^{2}-a y+1$. Denoting the other zero of this polynomial by $\varepsilon^{\prime}$, we get

$$
\begin{gathered}
\varepsilon=\frac{a+\sqrt{a^{2}-4}}{2} \text { and } \alpha=\frac{\varepsilon+\sqrt{\varepsilon^{2}+4}}{2}>\varepsilon, \\
\varepsilon^{\prime}=\frac{a-\sqrt{a^{2}-4}}{2} \text { and } \beta=\frac{\varepsilon^{\prime}+\sqrt{\varepsilon^{\prime 2}+4}}{2}<1+\varepsilon^{\prime} .
\end{gathered}
$$

We conclude that the roots of $f_{a}(x, 1)=0$ are $\alpha, \beta,-1 / \alpha,-1 / \beta$, and it is obvious that for $a \geq 3$ we have $\alpha \in(a-1, a), \beta \in(1,2)$, and $-1 / \alpha,-1 / \beta \in$ $(-1,0)$.

Similarly, the zeros of $g_{a}(x, 1)$ are $\alpha_{1}, \beta_{1},-1 / \alpha_{1},-1 / \beta_{1}$, with

$$
\begin{aligned}
& \varepsilon_{1}=\frac{a+\sqrt{a^{2}+4}}{2} \text { and } \alpha_{1}=\frac{\varepsilon_{1}+\sqrt{\varepsilon_{1}^{2}+4}}{2}, \\
& \varepsilon_{1}^{\prime}=\frac{a-\sqrt{a^{2}+4}}{2} \text { and } \beta_{1}=\frac{\varepsilon_{1}^{\prime}+\sqrt{\varepsilon_{1}^{\prime 2}+4}}{2} .
\end{aligned}
$$

If $a>1$, then $\alpha_{1} \in(a, a+1), \beta_{1} \in(0,1),-1 / \alpha_{1} \in(-1,0)$, and $-1 / \beta_{1} \in$ $(-2,-1)$, while $\alpha_{1} \in(2,3)$ for $a=1$.

\section{Fundamental units in the ORder $Z[\varphi]$}

The aim of this section is to find a system of fundamental units in the order $\mathbf{Z}[\varphi]$, where $\varphi$ is one of the zeros of $f_{a}(x, 1)$ or $g_{a}(x, 1)$. The result will be proved only in the first case because the proof of the second one is essentially the same.

Theorem 3.1. Let $\varphi$ be one of the roots of $f_{a}(x, 1)=0$. If $a=3$, then $\varphi-1, \varphi,(\varphi-1 / \varphi)^{1 / 2}$ and, if $a>3$, then $\varphi-1, \varphi, \varphi+1$ is a system of fundamental units of the order $\mathbf{Z}[\varphi]$.

Theorem 3.1a. Let $\psi$ be one of the roots of $g_{a}(x, 1)=0$ and $a>0$. Then $\psi-1, \psi, \psi+1$ is a system of fundamental units of the order $\mathbf{Z}[\psi]$.

Remark. The group of units $\langle\varphi-1, \varphi, \varphi+1\rangle$ as well as $\langle\psi-1, \psi, \psi+1\rangle$ does not coincide with the unit group of the maximal order of $\mathbf{Q}(\varphi)$ and $\mathbf{Q}(\psi)$, respectively, as one can see in the example $a=6$ in the first case, and $a=4$ in the second one. But to prove our main theorems, we need exactly the results stated above. Before proving Theorem 3.1, we establish three lemmas. 
Lemma 3.2. Let $\varepsilon>1$ be a unit in a real quadratic number field. Then $\varepsilon$ is a fundamental unit in $\mathrm{Z}[\varepsilon]$, except when $\varepsilon=(3+\sqrt{5}) / 2=((1+\sqrt{5}) / 2)^{2}$.

Proof. Assume that there exists $\omega \in \mathbf{Z}[\varepsilon]$ and an integer $k$ with $|k| \neq 0,1$ such that

$$
\varepsilon=\omega^{k} \text {. }
$$

As $\varepsilon>1$, replacing $\omega$ by $\omega^{-1}$ if necessary, we may assume $\omega>1$ and $k>1$. There exist integers $x_{n}, y_{n}$, for any $n \geq 0$, with

$$
\omega^{n}=x_{n}+y_{n} \varepsilon .
$$

Denote by $\gamma^{\prime}$ the conjugate of the element $\gamma \in \mathbf{Q}(\varepsilon)$. Then

$$
\omega^{\prime n}=x_{n}+y_{n} \varepsilon^{\prime},
$$

and so

$$
y_{n}=\frac{\omega^{n}-\omega^{\prime n}}{\varepsilon-\varepsilon^{\prime}}=y_{1} \frac{\omega^{n}-\omega^{\prime n}}{\omega-\omega^{\prime}} .
$$

Here we used $\omega-\omega^{\prime}=y_{1}\left(\varepsilon-\varepsilon^{\prime}\right)$, hence $y_{1}>0$. We have $\left(\omega^{n}-\omega^{\prime n}\right) /\left(\omega-\omega^{\prime}\right) \epsilon$ $\mathbf{Z}$ for any $n \geq 0$ because $\omega$ is a quadratic algebraic integer. Hence, if there exists a $k>1$ with (3.1), then $y_{1}=1$, and so

$$
y_{n}=\frac{\omega^{n}-\omega^{\prime n}}{\omega-\omega^{\prime}}
$$

Therefore, the sequence $\left\{y_{n}\right\}_{n=0}^{\infty}$ satisfies the recursion

$$
y_{n+1}=\left(\omega+\omega^{\prime}\right) y_{n}-\left(\omega \omega^{\prime}\right) y_{n-1},
$$

where $\omega+\omega^{\prime} \in \mathbf{Z}$ and $\left|\omega \omega^{\prime}\right|=1$.

If $\omega \omega^{\prime}=1$, then $\omega+\omega^{\prime} \geq 3$, and so $y_{n+1} \geq 2 y_{n} \geq 2^{n}$, which means that (3.1) cannot hold in this case.

If $\omega \omega^{\prime}=-1$, then again by $(3.3)$

$$
y_{n+1}>\left(\omega+\omega^{\prime}\right) y_{2} \geq y_{2}=\omega+\omega^{\prime} \geq 1,
$$

hence (3.1) can hold in this case only if $k=2$ and $\omega+\omega^{\prime}=1$. Then $\omega=$ $(1+\sqrt{5}) / 2$ and $\varepsilon=(3+\sqrt{5}) / 2=\omega^{2}$, which corresponds to the case $a=3$. The lemma is proved.

Lemma 3.3. Let $\varphi$ be a root of $f_{a}(x, 1)=0$ and $\varepsilon=\varphi-1 / \varphi$. Then $\mathbf{Z}[\varepsilon]=$ $\mathbf{Q}(\varepsilon) \cap \mathbf{Z}[\varphi]$.

Proof. Since

$$
\varepsilon=\varphi-\frac{1}{\varphi}=\varphi+(\varphi-1)(\varphi+1)(\varphi-a)=\varphi^{3}-a \varphi^{2}+a \in \mathbf{Z}[\varphi],
$$

we have $\mathbf{Z}[\varepsilon] \subseteq \mathbf{Q}(\varepsilon) \cap \mathbf{Z}[\varphi]$.

Let $\gamma \in \mathbf{Q}(\varepsilon) \cap \mathbf{Z}[\varphi]$. Then there exist $b_{i} \in \mathbf{Z}(i=0, \ldots, 3)$ and $a_{0}, a_{1} \in \mathbf{Q}$ such that

$$
a_{0}+a_{1} \varepsilon=b_{0}+b_{1} \varphi+b_{2} \varphi^{2}+b_{3} \varphi^{3} .
$$


Using (3.4), we get

$$
a_{0}+a a_{1}-a a_{1} \varphi^{2}+a_{1} \varphi^{3}=b_{0}+b_{1} \varphi+b_{2} \varphi^{2}+b_{3} \varphi^{3},
$$

which implies $a_{0}, a_{1} \in \mathbf{Z}$ immediately.

As in $\S 2$, let us denote by $\alpha$ the largest real root of $f_{a}(x, 1)=0$. In the sequel we order the conjugates of $\alpha$ so that $\alpha^{(1)}=\alpha, \alpha^{(2)}=\sigma(\alpha)=-1 / \alpha$, $\alpha^{(3)}=\beta, \alpha^{(4)}=\sigma(\beta)=-1 / \beta$. This implies the ordering of the conjugates for any $\partial \in \mathbf{Q}(\alpha)=\mathbf{K}$.

Lemma 3.4. Let $\omega \in \mathbf{Z}[\alpha]$ be such that $\omega^{(i)}>0, i=1, \ldots, 4$, and

$$
\omega^{(1)} \omega^{(2)}=\omega^{(3)} \omega^{(4)}=1 \text {. }
$$

Further, let

$$
W_{n}=\omega^{(1) n}+\omega^{(2) n}=b_{n} \varepsilon+c_{n}
$$

for any $n \geq 0$, where $b_{n}, c_{n} \in \mathbf{Z}$. Then we have

$$
b_{2 n}=b_{n}\left(\omega^{(1) n}+\omega^{(2) n}+\omega^{(3) n}+\omega^{(4) n}\right)
$$

for any $n>0$. Moreover, if

$$
\max \left\{\omega^{(i)} \mid i=1, \ldots, 4\right\}=\left\{\begin{array}{l}
\max \left\{\omega^{(3)}, \omega^{(4)}\right\} \\
\max \left\{\omega^{(1)}, \omega^{(2)}\right\},
\end{array}\right.
$$

then $b_{n}<0$ in the first case, and $b_{n}>0$ in the second, for any $n>0$.

Proof. It is clear from Lemma 3.3 that $W_{n} \in \mathbf{Z}[\varepsilon]$, hence (3.6) holds.

Denote by $\Psi: \mathbf{K}=\mathbf{Q}(\alpha) \rightarrow \mathbf{Q}(\beta)$ the isomorphism defined by $\Psi(\alpha)=\beta$. Then we have $\Psi\left(\gamma^{(1)}\right)=\gamma^{(3)}$ and $\Psi\left(\gamma^{(2)}\right)=\gamma^{(4)}$ for any $\gamma \in \mathbf{K}$. Furthermore, $\Psi(\varepsilon)=\varepsilon^{\prime}$.

Using these properties of $\Psi$, we get

$$
\Psi\left(W_{n}\right)=\omega^{(3) n}+\omega^{(4) n}=b_{n} \varepsilon^{\prime}+c_{n} .
$$

Subtracting (3.8) from (3.6), the following analytical formulae can be derived for $b_{n}$ :

$$
b_{n}=\frac{\omega^{(1) n}+\omega^{(2) n}-\omega^{(3) n}-\omega^{(4) n}}{\varepsilon-\varepsilon^{\prime}} .
$$

Using (3.5), we get

$$
b_{2 n}=\frac{W_{n}^{2}-\Psi\left(W_{n}\right)^{2}}{\varepsilon-\varepsilon^{\prime}}=b_{n}\left(W_{n}+\Psi\left(W_{n}\right)\right),
$$

which is exactly (3.7).

If $\max \left\{\omega^{(i)} \mid i=1, \ldots, 4\right\}=\max \left\{\omega^{(3)}, \omega^{(4)}\right\}$, then by (3.9) there exists an $n_{0}$ such that $b_{n}<0$ for any $n \geq n_{0}$. By assumption, $W_{n}+\Psi\left(W_{n}\right)>0$ holds for any $n$, hence (3.7) implies that $b_{n}<0$ for any $n>0$. The proof of the other case is exactly the same. 
Proof of Theorem 3.1. Without loss of generality we may assume $\varphi=\alpha$. Let $\varepsilon=\alpha-1 / \alpha=(\alpha-1)(\alpha+1) / \alpha$. Then $\alpha-1, \alpha, \alpha+1$ is a system of fundamental units if and only if $\alpha-1, \alpha, \varepsilon$ is. To prove this, it is enough to see by [8, Theorem (7.1), Chapter 5, p. 368] that none of the equations

$$
\begin{gathered}
\alpha=\omega^{k}, \\
\alpha-1=\alpha^{h} \omega^{k}, \\
\varepsilon=(\alpha-1)^{l} \alpha^{h} \omega^{k}
\end{gathered}
$$

is solvable, where $\omega \in Z[\alpha]$ and $0<l, h<k, k \geq 2$ are integers. For $\gamma \in \mathbf{Q}(\alpha)$ let $\bar{\gamma}=\sigma(\gamma)$, where $\sigma$ denotes the automorphism defined in Lemma 2.1 .

(a) Assume that (3.10) is solvable with $k>1$. Then we may assume $\omega>1$. From (3.5) we get $-1=\alpha \bar{\alpha}=(\omega \bar{\omega})^{k}$, hence $k$ is odd, $\omega \bar{\omega}=-1$, and $0>\bar{\omega}$.

On the other hand,

$$
\varepsilon=\alpha+\bar{\alpha}=\omega^{k}+\bar{\omega}^{k}=(\omega+\bar{\omega}) \frac{\omega^{k}+\bar{\omega}^{k}}{\omega+\bar{\omega}}>\omega+\bar{\omega} .
$$

Both numbers $\omega+\bar{\omega}$ and $\left(\omega^{k}+\bar{\omega}^{k}\right) /(\omega+\bar{\omega})$ belong to $\mathbf{Z}[\alpha]$, and $\varepsilon$ is a unit, hence $\omega+\bar{\omega}$ is a unit, too. Furthermore, $\overline{\omega+\bar{\omega}}=\omega+\bar{\omega}$, so $\omega+\bar{\omega} \in \mathbf{Q}(\varepsilon)$, hence $\omega+\bar{\omega} \in \mathbf{Z}[\varepsilon]$ by Lemma 3.3. By Lemma 3.2 there exists an integer $h \geq 1$ such that $\omega+\bar{\omega}=\varepsilon^{h}$, which contradicts (3.13).

(b) If (3.11) were solvable with $k>1$, then

$$
-\frac{(\alpha-1)(\alpha+1)}{\alpha}=(\alpha-1) \overline{(\alpha-1)}=(-1)^{h}(\omega \bar{\omega})^{k}
$$

would be satisfied, i.e., $\varepsilon= \pm(\omega \bar{\omega})^{k}$. But $\omega \bar{\omega}$ is a unit in $\mathbf{Z}[\varepsilon]$, which contradicts Lemma 3.2.

(c) Finally, assume that (3.12) is solvable with $k>1$. Let us first make the additional assumption that $h=l=0$. Then $\varepsilon=\omega^{(1) k}=\omega^{(2) k}$ and $\varepsilon^{\prime}=$ $\omega^{(3) k}=\omega^{(4) k}$, i.e., $\omega^{(1)}= \pm \omega^{(2)}$ and $\omega^{(3)}= \pm \omega^{(4)}$ because $\mathbf{K}$ is real. Lemma 3.2 implies $k=2, \omega^{(1)} \omega^{(2)}=-\varepsilon$, and $\omega^{(3)} \omega^{(4)}=-\varepsilon^{\prime}$. We may assume without loss of generality that $\omega^{(1)}=-\omega^{(2)}=\sqrt{\varepsilon}$ and $\omega^{(3)}=-\omega^{(4)}=\sqrt{\varepsilon}$. Then we have

$$
\left(\omega^{(1)}+\omega^{(3)}\right)^{2}=\omega^{(1)^{2}}+\omega^{(3)^{2}}+2=\varepsilon+\varepsilon^{\prime}+2=a+2,
$$

i.e., $\omega^{(1)}+\omega^{(3)}=\sqrt{a+2}$. One can derive $\omega^{(1)}+\omega^{(4)}=\sqrt{a-2}$ and $\omega^{(2)}+\omega^{(3)}=$ $-\sqrt{a-2}$ similarly.

The splitting field of $f_{a}(x, 1)$ has an automorphism given either by the permutation $(\beta,-1 / \beta)$ or by $(\alpha, \beta,-1 / \alpha,-1 / \beta)$. This automorphism maps either $\omega^{(1)}$ to $\omega^{(1)}$ and $\omega^{(3)}$ to $\omega^{(4)}$, hence $\sqrt{a+2}$ to $\sqrt{a-2}$, or $\omega^{(1)}$ to $\omega^{(3)}$ and $\omega^{(3)}$ to $\omega^{(2)}$, hence $\sqrt{a+2}$ to $-\sqrt{a-2}$, which is impossible. 
If $l=0$ and $h>0$, then we get

$$
\varepsilon^{2}=(-1)^{h}\left(\omega^{(1)} \omega^{(2)}\right)^{k}
$$

from (3.12). This implies $k=2, h=1$ by Lemma 2, which is obviously impossible. So we may assume in the sequel that $l, h>0$.

Considering the product of the first and second conjugates of (3.12), we get $l=2$ and $\omega^{(1)} \omega^{(2)}= \pm 1$ for the possible solutions. Hence $k$ is odd.

If $h$ is odd, too, then replacing $\omega$ by $\omega \alpha$, we may rewrite (3.12) as

$$
\varepsilon=(\alpha-1)^{2} \alpha^{h-k} \omega^{k},
$$

where $h-k$ is even and $-k<h-k$. So it is enough to prove that (3.12) is unsolvable in integers $l, h, k$ with $l=2,-k<h<k, h$ even, $k \geq 3$ odd.

Assume on the contrary that it is solvable. Then considering the sign of the conjugates of (3.12), we get

$$
\omega^{(1)}, \omega^{(2)}, \omega^{(3)}, \omega^{(4)}>0
$$

and

$$
\omega^{(1)} \omega^{(2)}=\omega^{(3)} \omega^{(4)}=1 .
$$

It follows from (3.12) that

$$
\omega^{(1) k}=\frac{\alpha+1}{(\alpha-1) \alpha} \frac{1}{\alpha^{h}}<\frac{1}{\alpha^{h}}
$$

and

$$
\omega^{(2) k}=\frac{\alpha-1}{\alpha+1} \alpha^{h+1}<\alpha^{h+1} .
$$

As $\alpha<a$, these inequalities imply

$$
\omega^{(1)}<\left\{\begin{array}{ll}
1 & \text { if } h \geq 0, \\
a & \text { if } h<0
\end{array} \quad \text { and } \quad \omega^{(2)}< \begin{cases}a & \text { if } h \geq 0, \\
1 & \text { if } h<0\end{cases}\right.
$$

Using (3.15), we get

$$
1<\omega^{(1)}+\omega^{(2)}<a+1 .
$$

We shall now show that if $h=0$ or -2 , then

$$
\max \left\{\omega^{(i)} \mid i=1, \ldots, 4\right\}=\omega^{(3)},
$$

and if $h$ is even and $h \neq 0,-2$, then

$$
\max \left\{\omega^{(1)}, \omega^{(2)}\right\}>\max \left\{\omega^{(3)}, \omega^{(4)}\right\}
$$

holds.

If $h \geq 0$, then $\omega^{(2) k}>1>\omega^{(1) k}$ by (3.16) and (3.17). Simple calculation shows that

$$
\left(\frac{\omega^{(2)}}{\omega^{(3)}}\right)^{k}=\left[(\alpha-1)(\beta-1) \alpha^{h / 2} \beta^{h / 2}\right]^{2}
$$


By (2.1) and (2.2) we have

$$
(\alpha-1)(\beta-1)<\frac{a-1}{\varepsilon}<1 .
$$

Hence, if $h=0$, then $\omega^{(2)}<\omega^{(3)}$, which proves (3.19). Otherwise, if $h>0$, then as $a \geq 4$ we have

$$
\alpha \beta(\alpha-1)(\beta-1)>\frac{\alpha(\alpha-1) \varepsilon^{\prime}}{2}=\frac{\alpha^{2}}{2(\alpha+1)}>1,
$$

and so $\omega^{(3)}<\omega^{(2)}$.

Similarly,

$$
\left(\frac{\omega^{(2)}}{\omega^{(4)}}\right)^{k}=\left[\frac{(\alpha-1)(\beta+1) \alpha^{h / 2}}{\beta^{(h+2) / 2}}\right]^{2},
$$

and as the inequality

$$
\left(\frac{\alpha}{\beta}\right)^{h / 2} \frac{(\alpha-1)(\beta+1)}{\beta}>1
$$

is obviously true, we have $\omega^{(2)}>\omega^{(4)}$. This proves $(3.20)$ for $h>0$. The case $h<0$ can be settled similarly.

Put $B_{n}=\omega^{(1) n}+\omega^{(2) n}$ for any $n \geq 0$. As we have shown in Lemma 3.4, there exist integers $b_{n}, c_{n} \in \mathbf{Z}$ with $B_{n}=b_{n} \varepsilon+c_{n}$.

Assume first that $h \neq 0,-2$. Then (3.20) holds and so $b_{n}>0$ for any $n>0$ by Lemma 3.4 . The second inequality (3.18) implies $c_{1} \leq 0$ immediately. Since by $(3.15)$

$$
\Psi\left(B_{n}\right)=\omega^{(3) n}+\omega^{(4) n}=b_{n} \varepsilon^{\prime}+c_{n}>1
$$

holds for any $n>0$, we have $b_{1}>\left(1-c_{1}\right) \varepsilon$. Using this again in (3.18), we get

$$
a+1>b_{1} \varepsilon+c_{1}>\left(1-c_{1}\right) \varepsilon^{2}+c_{1}=-c_{1}\left(\varepsilon^{2}-1\right)+\varepsilon^{2} \geq \varepsilon^{2},
$$

which is impossible. Hence (3.12) cannot hold if $h \neq 0,-2$.

Finally, assume that $h=0$ or $h=-2$. For $h=0$ and $h=-2$ we get

$$
B_{k}=\frac{\alpha+1}{(\alpha-1) \alpha}+\frac{(\alpha-1) \alpha}{\alpha+1}=-3 \varepsilon+4 a-2
$$

and

$$
B_{k}=\frac{(\alpha+1) \alpha}{\alpha-1}+\frac{\alpha-1}{(\alpha+1) \alpha}=-3 \varepsilon+4 a+2
$$

respectively from (3.16) and (3.17).

Hence, as $1, \varepsilon$ is a basis of the module $\mathbf{Z}[\varepsilon], b_{k}=-3$ must hold with $k \geq 3$.

We have seen that in these cases (3.17) holds, thus $b_{1}<0$ by Lemma 3.4. From the first inequality (3.18) we get $c_{1}>1-b_{1} \varepsilon$, which implies that

$$
\omega^{(1)}+\cdots+\omega^{(4)}=b_{1} a+2 c_{1}>-b_{1}(2 \varepsilon-a)+2>4 \text {. }
$$


Hence, $b_{2}<-4$ by (3.7), and as the sequence $b_{n}$ is monotonically decreasing, $b_{n}<-4$ for any $n \geq 2$. Hence, $b_{k}=-3$ for $k \geq 3$ cannot hold. Theorem 3.1 is proved.

\section{APPROXIMATION PROPERTIES OF THE SOlUtions OF (1.2)}

Let $(x, y) \in \mathbf{Z}^{2}$ be a solution of (1.2). If $x=0$, then $y= \pm 1$, and if $y=0$, then $x= \pm 1$. Furthermore, the pairs $(x, y)$ and $(-x,-y)$ are both solutions or are not solutions of (1.2), hence we assume in the sequel that $x y \neq 0$ and $y>0$.

Let $(x, y) \in \mathbf{Z}^{2}$ be a solution of (1.2), and $\gamma=x-\alpha y$. Then $\gamma \in \mathbf{Z}[\alpha]$, and we can reformulate (1.2) as

$$
f_{a}(x, y)=N_{K / Q}(\gamma)= \pm 1
$$

where $N_{K / Q}(\gamma)=\gamma^{(1)} \gamma^{(2)} \gamma^{(3)} \gamma^{(4)}$ denotes the norm of the element $\gamma$. The last equation means that $\gamma$ is a unit in the order $\mathbf{Z}[\alpha]$. Hence, if $a>3$, then there exist integers $a_{0}, a_{1}, a_{2}, a_{3}$ such that

$$
\gamma=(-1)^{a_{0}}(\alpha-1)^{a_{1}} \alpha^{a_{2}}(\alpha+1)^{a_{3}} \text {. }
$$

The norm of each of the numbers $(-1), \alpha-1, \alpha$, and $\alpha+1$ is 1 , hence on the right-hand side of $(4.1)$ only the + sign is possible. This proves already the first assertion of the main theorem if $a>3$. For $a=3$, replacing $\alpha+1$ with $(\alpha-1 / \alpha)^{1 / 2}$, the above considerations remain valid.

In the remaining of this section we prove that if $(x, y) \in \mathbf{Z}^{2}$ is a solution of (1.2) with $y \neq 0$, then $x / y$ is a good approximation of one of the conjugates of $\alpha$. Hence, we can divide the solutions into four distinct classes, and it is enough to examine only two of them.

Lemma 4.1. Let $(x, y) \in \mathbf{Z}^{2}$ be a solution of (1.2). Then $(-y, x)$ is also a solution. Furthermore, if $y \neq 0$, then

$$
-1 \leq x / y \leq a .
$$

Proof. The polynomial $f_{a}(x, y)$ satisfies the identity $f_{a}(x, y)=f_{a}(-y, x)$, from which the first assertion follows at once.

If $y \neq 0$, then we get

$$
y^{4}\left(\frac{x}{y}-\alpha\right)\left(\frac{x}{y}+\frac{1}{\alpha}\right)\left(\frac{x}{y}-\beta\right)\left(\frac{x}{y}+\frac{1}{\beta}\right)=y^{4} f_{a}\left(\frac{x}{y}, 1\right)=1
$$

from (4.1). The inequality $x / y<-1$ implies

$$
\begin{aligned}
1 & =y^{4} f_{a}\left(\frac{x}{y}, 1\right)>y^{4}(-1-\alpha)\left(-1+\frac{1}{\alpha}\right)(-1-\beta)\left(-1+\frac{1}{\beta}\right) \\
& =y^{4} N_{K / Q}(\alpha+1)=y^{4},
\end{aligned}
$$

which is impossible. One can get a similar contradiction assuming $x / y>a$. The lemma is proved. 
Let $(x, y) \in \mathbf{Z}^{2}$ be a solution of $(1.2)$ with $y \neq 0$. As $(-x,-y)$ and $(-y, x)$ are also solutions, we may choose $x>0$ and $y>0$. Clearly,

$$
\frac{x}{y}-\alpha<\frac{x}{y}-\beta<\frac{x}{y}+\frac{1}{\alpha}<\frac{x}{y}+\frac{1}{\beta} \text {. }
$$

So, either $x / y-\alpha>0$ and then $x / y-\alpha=\min _{1 \leq i \leq 4}\left\{x / y-\alpha^{(i)}\right\}$, which is the characterization of a Type I solution, or $x / y-\alpha<0$ and then $x / y-\beta<0$, as the product $\prod_{i=1}^{4}\left(x / y-\alpha^{(i)}\right)$ is positive. But then

$$
0<\beta-\frac{x}{y}<\min \left\{\alpha-\frac{x}{y}, \frac{x}{y}+\frac{1}{\alpha}, \frac{x}{y}+\frac{1}{\beta}\right\},
$$

which is the characterization of a Type II solution.

\section{Solutions OF TyPe I}

Throughout this section, a solution of (1.2) means a solution of Type I. Let $\varepsilon=\alpha-1 / \alpha$ and $\varepsilon^{\prime}=\beta-1 / \beta$ as in $\S 3$. We assume, furthermore, $a>3$.

Lemma 5.1. Let $(x, y) \in \mathbf{Z}^{2}$ be a solution of (1.2) and put $\gamma=x-\alpha y$. Further, let $a_{0} \in\{0,1\}$ and let $\left(a_{1}, a_{2}, a_{3}\right) \in \mathbf{Z}^{3}$ be as in (4.2). Then $a_{0}=0, a_{1}+a_{2}=$ $u$ is even, $a_{1}+a_{3}=-v<-1$,

$$
x y=\frac{\varepsilon^{v}-\varepsilon^{\prime v}}{\varepsilon-\varepsilon^{\prime}},
$$

and

$$
x^{2}-y^{2}=\frac{\varepsilon^{v+1}-\varepsilon^{v+1}}{\varepsilon-\varepsilon^{\prime}} .
$$

Proof. The solution is of Type I, hence $\gamma>0$ and $a_{0}=0$. The equation (4.2) implies

$$
\gamma \sigma(\gamma)=(-1)^{u} \varepsilon^{-v}=x^{2}-x y \varepsilon-y^{2}>0,
$$

hence $u$ is even. Further, we have $\left(x^{2}-x y \varepsilon-y^{2}\right)\left(x^{2}-x y \varepsilon^{\prime}-y^{2}\right)=1$ and $\left(x^{2}-x y \varepsilon^{\prime}-y^{2}\right)=(x-\beta y)(x+y / \beta)>1$, hence $x^{2}-x y \varepsilon-y^{2}<1$, and so $v>0$. Therefore,

$$
x^{2}-x y \varepsilon-y^{2}=\varepsilon^{\prime v}
$$

and

$$
x^{2}-x y \varepsilon^{\prime}-y^{2}=\varepsilon^{v}
$$

which imply (5.1) and (5.2) immediately.

If $v=1$, then $(5.1)$ implies $x y=1$, hence $x / y=1$, but then $(x, y)$ is a solution of Type II. The lemma is proved.

Corollary 5.2. Under the same assumptions as in Lemma 5.1 we have

$$
\frac{\varepsilon^{v / 2}}{a}<y<\frac{\varepsilon^{v / 2}}{a-1}
$$


Proof. We have $a-1<a<x / y \leq a$ by Lemma 4.1, and so (5.1) implies

$$
(a-1) y^{2}<\frac{\varepsilon^{v}-\varepsilon^{\prime v}}{\varepsilon-\varepsilon^{\prime}}<\frac{\varepsilon^{v}}{a-1},
$$

as $\varepsilon-\varepsilon^{\prime}=\sqrt{a^{2}-4}>a-1$, which proves the second inequality in (5.4).

We have $v \geq 2$ by Lemma 5.1. Under this assumption an easy computation shows that

$$
\frac{\varepsilon^{v}-\varepsilon^{\prime v}}{\varepsilon-\varepsilon^{\prime}}>\frac{\varepsilon^{v}}{a}
$$

This inequality, (4.3), and (5.1) imply the first inequality in (5.4).

Lemma 5.3. Let the assumptions be the same as in Lemma 5.1. Then we have

$$
\begin{gathered}
\frac{a-1}{a+1} \varepsilon^{-3 v / 2}<x-\alpha y<\varepsilon^{-3 v / 2}, \\
\frac{a-1}{a+1} \varepsilon^{v / 2}<x-\beta y<\varepsilon^{v / 2}, \\
\varepsilon^{v / 2}<x+\frac{1}{\beta} y<\frac{a+1}{a-1} \varepsilon^{v / 2}
\end{gathered}
$$

Proof. We know that $\alpha<x / y \leq a$ and $\alpha>1$. From $8 a^{2}>36$ it is easy to deduce that $\sqrt{\varepsilon^{2}+4}>a$. Combining these inequalities, we get

$$
a+1>\frac{x}{y}+\frac{1}{\alpha}>\alpha+\frac{1}{\alpha}=\sqrt{\varepsilon^{2}+4}>a .
$$

We can rewrite (5.3) as

$$
y\left(\frac{x}{y}+\frac{1}{\alpha}\right)(x-\alpha y)=\varepsilon^{v},
$$

which, together with (5.4) and (5.8) implies (5.5).

By $(5.3 \mathrm{a})$ and $\beta>1$ we have

$$
x-\beta y=\frac{1}{(x / y+1 / \beta) y} \varepsilon^{v} \geq \frac{1}{(a+1 / \beta) y} \varepsilon^{v}>\frac{a-1}{a+1} \varepsilon^{v / 2}
$$

and

$$
x-\beta y=\left(\frac{x}{y}-\beta\right) y \leq(a-\beta) y<\varepsilon^{v / 2},
$$

which proves (5.6).

We have finally

$$
a+1>\frac{x}{y}+\frac{1}{\beta}>\frac{x}{y}+\frac{1}{\alpha}>a .
$$

Combining this inequality with (5.4), one gets (5.7).

Lemma 5.4. Let the assumptions be the same as in Lemma 5.1. If $a_{1}=a_{3}$, then $a_{1}=-1$ and $x=a, y=1$.

Proof. If $a_{1}=a_{3}$, then $v$ is even, say $v=2 w$, with $w=-a_{1}$. For $n \geq 0$ put

$$
H_{n}=\frac{\varepsilon^{n}-\varepsilon^{\prime n}}{\varepsilon-\varepsilon^{\prime}} \text { and } S_{n}=\varepsilon^{n}+\varepsilon^{\prime n}
$$


Then $H_{n}, S_{n} \in \mathbf{Z}$ and $H_{2 n}=H_{n} S_{n}$. Using this notation, we have

$$
\begin{gathered}
x y=H_{2 w}=H_{w} S_{w}, \\
x^{2}-y^{2}=H_{2 w+1}=S_{w} H_{w+1}-1
\end{gathered}
$$

from (5.1) and (5.2)

Assume that $H_{w} \leq y<x \leq S_{w}$; then we get

$$
S_{w} H_{w+1}-1 \leq S_{w}^{2}-H_{w}^{2}
$$

by (5.10), i.e.,

$$
H_{w}^{2}-1 \leq S_{w}\left(S_{w}-H_{w+1}\right)
$$

We have

$$
S_{w}-H_{w+1}=\varepsilon^{w}+\varepsilon^{\prime w}-\frac{\varepsilon^{w+1}-\varepsilon^{\prime w+1}}{\varepsilon-\varepsilon^{\prime}}=\frac{\varepsilon^{\prime w-1}-\varepsilon^{w-1}}{\varepsilon-\varepsilon^{\prime}}=-H_{w-1} \leq 0,
$$

and equality holds if and only if $w=1$. On the other hand, $H_{w}^{2}-1 \geq 0$, and equality holds again if and only if $w=1$. Hence, $w=1$, and so $v=2$, which gives the solution described in the lemma. Otherwise, we have

$$
y<H_{w}<S_{w}<x .
$$

First we show that $w=2$ cannot give a solution. Indeed, in this case, $H_{2}=a$ and $S_{2}=a^{2}-2$, which imply $y \leq a-1$ and $x \geq a^{2}-1$ by (5.12), and so $x / y \geq a+1$, which is impossible by Lemma 2.1 . We may assume in the following $w \geq 3$.

From the proof of Lemma 5.3 and (5.12) we get

$$
x-\alpha y<\frac{1}{S_{w} \varepsilon^{2 w}}<\varepsilon^{-3 w}
$$

and

$$
x-\beta y>(\alpha-\beta) y>\frac{\alpha-\beta}{a} \varepsilon^{w} .
$$

On the other hand, (4.2) implies

$$
x-\alpha y=(\alpha-1)^{a_{1}} \alpha^{a_{2}}(\alpha+1)^{a_{3}}=\left(\frac{\alpha^{2}-1}{\alpha}\right)^{a_{1}} \alpha^{a_{1}+a_{2}}=\varepsilon^{a_{1}} \alpha^{a_{1}+a_{2}}
$$

and

$$
x-\beta y=\varepsilon^{\prime a_{1}} \beta^{a_{1}+a_{2}} .
$$

Using now (5.13) and (5.14), we get

$$
\frac{\log ((\alpha-\beta) / a)}{\log \beta}<a_{1}+a_{2}<2 a_{1} \frac{\log \varepsilon}{\log \alpha} .
$$

Hence,

$$
2 a_{1}>\frac{\log \alpha}{\log \varepsilon} \frac{\log ((\alpha-\beta) / a)}{\log \beta} .
$$


An easy computation shows that the right-hand side is larger than -6 , and so $a_{1}>-3$. Of course, as $\alpha-\beta>a-2$ and $\beta>1+1 / 2 \varepsilon$, we have

$$
\beta^{6}(\alpha-\beta)>\left(1+\frac{3}{a}\right)(a-2)>a
$$

if $a \geq 6$. For $3 \leq a<6$ direct calculation gives the same estimate. Hence, $\log (a /(\alpha-\beta)) / \log \beta<6$. The inequality $\log \alpha / \log \varepsilon<1$ is obvious, so the assertion is true.

We have seen that $a_{1}=-2$ is impossible, which proves the lemma.

Lemma 5.5. Let the assumptions be the same as in Lemma 5.1. Then

$$
a_{1}+a_{3}+2 a_{2}=-2 \frac{\log \varepsilon \log ((\beta+1) /(\beta-1))}{A} v+\partial_{2},
$$

$$
a_{3}-a_{1}=2 \frac{\log \varepsilon \log \beta}{A} v+\partial_{3},
$$

where $A=\log \alpha \log ((\beta+1) /(\beta-1))-\log \beta \log ((\alpha+1) /(\alpha-1)), \quad-1.26<\partial_{2}<$ 0.27 , and $-1<\partial_{3}<0.27$.

Proof. We get

$$
\begin{gathered}
\log \frac{a-1}{a+1}-\frac{3}{2} v \log \varepsilon<a_{1} \log (\alpha-1)+a_{2} \log \alpha+a_{3} \log (\alpha+1)<-\frac{3}{2} v \log \varepsilon, \\
\log \frac{a-1}{a+1}+\frac{v}{2} \log \varepsilon<a_{1} \log (\beta-1)+a_{2} \log \beta+a_{3} \log (\beta+1)<\frac{v}{2} \log \varepsilon, \\
a_{1}+a_{3}=-v
\end{gathered}
$$

from (4.2), (5.5), (5.6) and from the definition of $v$. This system of inequalities implies at once

$$
\begin{gathered}
\log \frac{a-1}{a+1} \log \beta<-A a_{3}-V_{1} v<-\log \frac{a-1}{a+1} \log \alpha, \\
\log \frac{a-1}{a+1} \log \frac{\beta+1}{\beta-1}<A a_{2}-V_{2} v<-\log \frac{a-1}{a+1} \log \frac{\alpha+1}{\alpha-1},
\end{gathered}
$$

where

$$
V_{1}=\log (\alpha-1) \log \beta-\frac{3}{2} \log \varepsilon \log \beta-\log (\beta-1) \log \alpha-\frac{1}{2} \log \varepsilon \log \alpha
$$

and

$$
\begin{aligned}
V_{2}= & \log (\alpha-1) \log \frac{\beta+1}{\beta-1}-\frac{3}{2} \log \varepsilon \log \frac{\beta+1}{\beta-1} \\
& -\log (\beta-1) \log \frac{\alpha+1}{\alpha-1}-\frac{1}{2} \log \varepsilon \log \frac{\alpha+1}{\alpha-1} .
\end{aligned}
$$

Elementary computations show that

$$
V_{1}=\frac{1}{2} A-\log \varepsilon \log \beta, \quad V_{2}=\frac{1}{2} A-\log \varepsilon \log \frac{\beta+1}{\beta-1} .
$$


Hence,

$$
\begin{aligned}
a_{1}+a_{3}+2 a_{2} & =-v+2\left(\frac{1}{2}-\frac{\log \varepsilon \log ((\beta+1) /(\beta-1))}{A}\right) v+\partial_{2} \\
& =-2 \frac{\log \varepsilon \log ((\beta+1) /(\beta-1))}{A} v+\partial_{2},
\end{aligned}
$$

where

$$
\frac{2}{A} \log \frac{a-1}{a+1} \log \frac{\beta+1}{\beta-1}<\partial_{2}<\frac{2}{A} \log 2 \log \frac{\alpha+1}{\alpha-1} .
$$

This implies that if $a \geq 4$ then $-1.26<\partial_{2}<0.27$.

Similarly,

$$
a_{3}-a_{1}=v+2 a_{3}=v+2\left(-\frac{1}{2}+\frac{\log \varepsilon \log \beta}{A}\right) v+\partial_{3}=2 \frac{\log \varepsilon \log \beta}{A} v+\partial_{3},
$$

where

$$
\frac{2}{A}\left[\log \frac{a-1}{a+1} \log \alpha\right]<\partial_{3}<\frac{2}{A} \log \frac{a+1}{a-1} \log \beta,
$$

hence $-1<\partial_{3}<0.27$.

Lemma 5.6. Let the assumptions be the same as in Lemma 5.1 and put

$$
\Lambda_{1}=\log \frac{\alpha+1 / \beta}{\alpha-\beta}+\left(a_{1}+a_{3}+2 a_{2}\right) \log \beta+\left(a_{3}-a_{1}\right) \log \frac{\beta+1}{\beta-1} .
$$

Then

$$
0<\left|\Lambda_{1}\right|<3.1 \cdot \varepsilon^{-2 v-1} .
$$

Proof. Using the notations introduced at the beginning of $\S 4$, we get

$$
\left(\alpha+\frac{1}{\beta}\right) \gamma^{(3)}-(\alpha-\beta) \gamma^{(4)}=\left(\beta+\frac{1}{\beta}\right) \gamma^{(1)} \text {. }
$$

This implies by (5.5), (5.6), and (5.7) that

$$
0<\frac{\alpha+1 / \beta}{\alpha-\beta} \frac{\gamma^{(3)}}{\gamma^{(4)}}-1=\frac{\beta+1 / \beta}{\alpha-\beta} \frac{\gamma^{(1)}}{\gamma^{(4)}}<\frac{\sqrt{\varepsilon^{\prime 2}+4}}{\alpha-\beta} \frac{a}{a-1} \varepsilon^{-2 v}<3 \cdot \varepsilon^{-2 v-1} .
$$

If $v \geq 1$, then $3 \varepsilon^{-2 v-1}<0.06$, and using Lemma 2.2 of de Weger [14], we get

$$
\left|\log \frac{\alpha+1 / \beta}{\alpha-\beta}+\log \gamma^{(3)}-\log \gamma^{(4)}\right|<3.1 \cdot \varepsilon^{-2 v-1},
$$

which is equivalent to (5.17) by virtue of (4.2).

Lemma 5.7. Besides the assumptions of Lemma 5.1 suppose that $a_{1} \neq a_{3}$. Then

$$
v>\frac{1}{2} \varepsilon \log \varepsilon \text {. }
$$

Proof. By Lemma 5.1 and $a_{1} \neq a_{3}$ we have $u \geq 3$. Hence, $a_{1}+a_{3}+2 a_{2} \leq-5$ and $a_{3}-a_{1} \geq-1$ by (5.15) and (5.16).

Assume that $a_{3}-a_{1}=-1$. Then

$$
\Lambda_{1} \leq \log \frac{\alpha+1 / \beta}{(\beta+1)(\alpha-\beta)}+\log \frac{\beta-1}{\beta}-4 \log \beta<-4 \log \beta .
$$


On the other hand, $\beta>1+\varepsilon^{\prime} / 2$, hence $\beta^{2}>1+\varepsilon^{\prime}$, and so $4 \log \beta>\varepsilon^{-1}$ in contradiction with (5.17). Hence $a_{3}-a_{1} \geq 1$. In this case, the first and the third summand of $\Lambda_{1}$ are positive while the second is negative. Since $\log ((\alpha+1 / \beta) /(\alpha-\beta))>3.1 \varepsilon^{-2 v-1},(5.17)$ can hold only if

$$
\left(a_{1}+a_{3}+2 a_{2}\right) \log \beta+\left(a_{3}-a_{1}\right) \log \frac{\beta+1}{\beta-1}<0,
$$

i.e.,

$$
-\left(a_{1}+a_{3}+2 a_{2}\right) \log \beta>\log \frac{1}{\beta-1}+\log 2 .
$$

Further, $\log \beta=\log (1+(\beta-1))<\beta-1$ because of $\beta-1<\frac{1}{2}$, hence

$$
-\left(a_{1}+a_{3}+2 a_{2}\right)>\frac{1}{\beta-1} \log \frac{1}{\beta-1}+\frac{1}{\beta-1} \log 2>\varepsilon \log \varepsilon+\varepsilon \log 2 .
$$

Here we also used (2.2). We have $-\left(a_{1}+a_{3}+2 a_{2}\right)<2 v+1.26$ by (5.15), which together with the last inequality implies (5.18).

Theorem 5.8. If $a>9.9 \cdot 10^{27}$, then (4.1) has no solution of Type I with $v>2$. Proof. We use Corollary 2 of Blass et al. [2] to get a lower bound for $\left|\Lambda_{1}\right|$. In the sequel, $h(\gamma)$ denotes the absolute logarithmic height of the algebraic number $\gamma$. Adapting the notations of the cited paper, we have $n=3, D=8$, and $h(\beta), h((\beta+1) /(\beta-1))<\frac{1}{2} \log a$, so we may take $V_{1}=V_{2}=\frac{1}{2} \log a$. Further,

$$
h\left(\frac{\alpha+1 / \beta}{\alpha-\beta}\right)<\frac{9}{2} \log a,
$$

hence $V_{3}=\frac{9}{2} \log a$ is suitable. If $a \geq 10$, then $V_{2}^{+}=V_{2}$ and $V_{3}^{+}=V_{3}$ are also satisfactory. It follows from (5.15) and (5.16) that $\left|a_{3}-a_{1}\right| \leq\left|a_{1}+a_{3}+2 a_{2}\right|<$ $2 v+1.26$, hence Corollary 2 of Blass et al. [2] implies that

$$
\begin{aligned}
\left|\Lambda_{1}\right|>\exp \left\{-2^{48} \cdot 8^{5} \cdot e^{6} \log ^{3} a \log \left(2^{40} 3^{3} \log ^{3} a\right)\right. \\
\left.\cdot[\log (2 v+1.26)+12 \log \log a+90](\log 4)^{-4}\right\} .
\end{aligned}
$$

If $a \geq 100$, then combining the last inequality with (5.17) we get

$$
(2 v+1) \log \varepsilon-\log 3.1<1.52 \cdot 2^{68} \log ^{3} a \log \left(2^{40} 3^{3} \log ^{3} a\right) \log (2 v+1.26) .
$$

For $a \geq 100,(5.18)$ implies

$$
(2 v+1) \log \varepsilon-\log 3.1>v \log a,
$$

hence

$$
v<1.52 \cdot 2^{68} \log ^{2} a \log \left(2^{40} \cdot 3^{3} \log ^{3} a\right) \log (2 v+1.26) .
$$

Let $H_{a}(x)=x-1.52 \cdot 2^{68} \log ^{2} a \log \left(2^{40} \cdot 3^{3} \log ^{3} a\right) \log (2 x+1.26)$. Let $M_{a}$ be the root of the equation $H_{a}(x)=0$ for fixed $a$. Then $v<M_{a}$. On the other hand, $v>a$ for $a \geq 100$ by (5.18). Hence, if $a_{0}$ denotes the root of the equation 
$H_{a}(a)=0$, then for $a>a_{0},(5.19)$ cannot hold. An easy computation shows that $a_{0}<1.5 \cdot 2^{92} \approx 9.9 \cdot 10^{27}$, which proves the theorem.

\section{SOLUTIONS OF TYPE II}

In this section we assume that $a>3$ and $(x, y) \in \mathbf{Z}^{2}$ is a solution of (1.1) of Type II. Let $\gamma=x-\alpha y$, and denote by $a_{0}=0$ or $1,\left(a_{1}, a_{2}, a_{3}\right) \in \mathbf{Z}^{3}$ the corresponding exponents determined by (4.2). We refer in this section to these assumptions as general assumptions.

Lemma 6.1. Under the general assumptions we have $a_{0}=0, a_{1}+a_{2}=u$ is odd, $a_{1}+a_{3}=v>0$,

$$
x y=\frac{\varepsilon^{v}-\varepsilon^{\prime v}}{\varepsilon-\varepsilon^{\prime}}
$$

and

$$
x^{2}-y^{2}=\frac{\varepsilon^{v-1}-\varepsilon^{\prime v-1}}{\varepsilon-\varepsilon^{\prime}} .
$$

Proof. The same as the proof of Lemma 5.1.

Corollary 6.2. Under the general assumptions we have

$$
\frac{\varepsilon^{v / 2}}{\sqrt{a}}<y<\frac{\varepsilon^{v / 2}}{\sqrt{a-1}} .
$$

Proof. The same as the proof of Corollary 5.2.

Lemma 6.3. Under the general assumptions we have

$$
\begin{gathered}
\frac{\sqrt{a-1}}{2} \varepsilon^{-3 v / 2}<\beta y-x<\varepsilon^{-(3 v-1) / 2}, \\
\frac{a-2}{\sqrt{a}} \varepsilon^{v / 2}<\alpha y-x<\sqrt{a-1} \varepsilon^{v / 2}, \\
\frac{a+1}{\sqrt{a}} \varepsilon^{v / 2}<x+\frac{1}{\alpha} y<\frac{a+1}{\sqrt{a-1}} \varepsilon^{v / 2} .
\end{gathered}
$$

Proof. Similar as the proof of Lemma 5.3.

Lemma 6.4. Besides the general assumptions, suppose $a_{1}+a_{3}+2 a_{2}=1$. Then $a_{2}=0$ and $\left(a_{1}, a_{3}\right)=(1,0)$ or $(0,1)$, which corresponds to the solution $x=y=1$.

Proof. Using the notation of Lemma 6.1, we get $v=1-2 a_{2}>0$, hence $a_{2} \leq 0$. Put $w=-a_{2}$; then $v=2 w+1$. With the notation of the proof of Lemma 5.4 we get

$$
\begin{gathered}
x y=H_{2 w+1}=S_{w} H_{w+1}-1, \\
x^{2}-y^{2}=H_{2 w}=S_{w} H_{w}
\end{gathered}
$$


from (6.1) and (6.2). For $w=0$ this system of equations implies the solutions asserted, and it is easy to see that for $w=1$ the system does not have any solutions. Hence, we may assume in the sequel $w \geq 2$, in which case $H_{w+1}>$ $S_{w}$.

We shall now prove that

$$
y \leq S_{w}<H_{w+1} \leq x .
$$

Assume that this is not true. Then by (6.7),

$$
S_{w} H_{w}=x^{2}-y^{2}<H_{w+1}^{2}-S_{w}^{2},
$$

i.e.,

$$
H_{w+1}^{2}>S_{w}\left(S_{w}+H_{w}\right)
$$

Using the definitions of $S_{w}, H_{w}, \varepsilon$, and $\varepsilon^{\prime}$, one can prove by an easy computation that

(6.10) $\left(S_{w}\left(S_{w}+H_{w}\right)-H_{w+1}^{2}\right)\left(\varepsilon-\varepsilon^{\prime}\right)^{2}=\left(a^{2}-2 a-2\right) S_{2 w-1}-(a-3) S_{2 w-2}+2 S_{2}-2$.

We have $a>2$, hence $a^{2}-2 a-2>a(a-3)$, and so the right-hand side of (6.10) is large, so that

$$
(a-3)\left(a S_{2 w-1}-S_{2 w-2}\right)+2\left(a^{2}-3\right)=(a-3) S_{2 w}+2\left(a^{2}-3\right)>0,
$$

which contradicts (6.9); thus, (6.8) is indeed true. Using this precise bound, we get

$$
\beta y-x<\frac{1}{x \varepsilon^{v}}<\varepsilon^{-(3 w+1)} .
$$

The assumption $a_{1}+a_{3}+2 a_{2}=1$ together with (4.2) imply now

$$
\left(\frac{\beta+1}{\beta-1}\right)^{a_{3}-a_{1}} \beta=\frac{\beta y-x}{y / \beta+x}<\varepsilon^{-(4 w+1)},
$$

hence

$$
a_{3}-a_{1}<-(4 w+1) \frac{\log \varepsilon}{\log ((\beta+1) /(\beta-1))} .
$$

Similarly,

$$
\alpha y-x=(\alpha-\beta) y+(\beta y-x)>(\alpha-\beta) y
$$

and

$$
\frac{1}{\alpha} y+x<\frac{\alpha \beta+1}{\alpha} y \text {. }
$$

Thus, by (4.2),

$$
\left(\frac{\alpha+1}{\alpha-1}\right)^{a_{3}-a_{1}} \alpha=\frac{\alpha y-x}{y / \alpha+x}>\frac{\alpha(\alpha-\beta)}{\alpha \beta+1}
$$

and so

$$
a_{3}-a_{1}>\frac{\log ((\alpha-\beta) /(\alpha \beta+1))}{\log ((\alpha+1) /(\alpha-1))} .
$$


Comparing this with $(6.11)$, we get

$$
4 w+1<\frac{\log ((\beta+1) /(\beta-1))}{\log \varepsilon} \frac{\log ((\alpha \beta+1) /(\alpha-\beta))}{\log ((\alpha+1) /(\alpha-1))} .
$$

Both factors on the right are at most 2 , hence $w<1$, and the lemma is proved.

Lemma 6.5. Suppose the general assumptions. If $a \geq 30$, then

$$
a_{1}+a_{3}+2 a_{2}=2 v \frac{\log \varepsilon \log ((\alpha+1) /(\alpha-1))}{A}+\partial_{2}
$$

and

$$
a_{3}-a_{1}=-2 v \frac{\log \varepsilon \log \alpha}{A}+\partial_{3},
$$

where $A=\log \alpha \log ((\beta+1) /(\beta-1))-\log \beta \log ((\alpha+1) /(\alpha-1)), 0.95<\partial_{2}<1$, and $0.4<\partial_{3}<1$.

Proof. The same as the proof of Lemma 5.5.

Lemma 6.6. Suppose the general assumptions, and let

$$
\Lambda_{2}=\log \frac{\beta+1 / \alpha}{\alpha-\beta}+\left(a_{1}+a_{3}+2 a_{2}\right) \log \alpha+\left(a_{3}-a_{1}\right) \log \frac{\alpha+1}{\alpha-1} .
$$

Then

$$
\left|\Lambda_{2}\right|<1.1 \cdot \varepsilon^{-2 v}
$$

Proof. On the basis of the identity

$$
\left(\beta+\frac{1}{\alpha}\right) \gamma^{(1)}-(\beta-\alpha) \gamma^{(2)}=\left(\alpha+\frac{1}{\alpha}\right) \gamma^{(3)},
$$

the proof is similar to that of Lemma 5.6.

Lemma 6.7. Suppose the general assumptions, $a_{1}+a_{3}+2 a_{2} \neq 1$, and $a \geq 30$. Then

$$
v>\frac{1}{5} \varepsilon \log \varepsilon .
$$

Proof. By Lemma 6.5 we have $a_{3}-a_{1} \leq 0$ and $a_{1}+a_{3}+2 a_{2}>0$. Assume $a_{3}-a_{1}=0 ;$ then $(6.13)$ implies

$$
v=\frac{A \partial_{3}}{2 \log \varepsilon \log \alpha}
$$

and so

$$
a_{1}+a_{3}+2 a_{2}=\partial_{3} \frac{\log ((\alpha+1) /(\alpha-1))}{\log \alpha}+\partial_{2}<2 .
$$

Hence, $a_{1}+a_{3}+2 a_{2}=1$, which is excluded in the assumptions. Therefore, $a_{1}-a_{3}>0$. The inequality (6.14) can hold only if

$$
\left(a_{1}+a_{3}+2 a_{2}-1\right) \log \alpha+\left(a_{3}-a_{1}\right) \log \frac{\alpha+1}{\alpha-1}<0
$$

because of $\log ((\alpha \beta+1) /(\alpha-\beta))>1.1 \cdot \varepsilon^{-4}$. 
We have $(\alpha+1) /(\alpha-1)=1+2 /(\alpha-1)$ and $2 /(\alpha-1)<\frac{2}{29}$, hence (6.16) implies that

$$
a_{1}-a_{3}>\frac{\alpha-1}{2.08} \log \alpha \text {. }
$$

Using now (6.13) completes the proof of the lemma.

Theorem 6.8. If $a>9.9 \cdot 10^{27}$, then (4.1) has no solutions of Type II with $v>1$. Proof. The same as the proof of Theorem 5.8, apart from working with $\Lambda_{2}$ instead of $\Lambda_{1}$.

\section{PROOF OF THE THEOREMS}

Proof of Theorem 1. At the beginning of $\S 4$ we proved the first assertion, while Theorems 5.8 and 6.8 together with Lemmas 4.2 and 4.3 establish the second.

Proof of Theorem 2. We cannot give here the proof in the classical sense, because it is a lengthy procedure on a computer. We rather describe the method of the computer search.

Assume first that $a>3$. Computing the solutions of Type I, we may assume by Lemmas 5.1 and 5.4 that $v>2$. Let $B_{1}=-\left(a_{1}+a_{3}+2 a_{2}\right)$ and $B_{2}=a_{3}-a_{1}$. Then we have

$$
2 v+1.26>B_{1}>B_{2} \geq 1
$$

by Lemma 5.5 and by the proof of Lemma 5.7. Applying now Corollary 2 of Blass et al. [2] to $\Lambda_{1}$, we get $B_{1}<10^{32}$ for $a \leq 100$. Performing for $4<a \leq 100$ a modified version of the reduction procedure of Baker and Davenport [1], implemented by Gaál and Schulte [3], we get that $B_{2}<6$. For the remaining small values of $v$ we can compute the solutions from (5.1) and (5.2).

The nontrivial solutions of Type II satisfy $v \geq 4$ by Lemmas 6.1 and 6.4. Let now $B_{1}=a_{1}+a_{3}+2 a_{2}$ and $B_{2}=-\left(a_{3}-a_{1}\right)$. Then computing for $4 \leq a<30$ lower and upper bounds for $\partial_{2}$ and $\partial_{3}$ (defined in Lemma 6.5), we get

$$
2 v>B_{2}>B_{1} \geq 1 \text {. }
$$

Applying again Corollary 2 of [2] to $\Lambda_{2}$, we get $B_{2}<10^{32}$, which can be reduced to $B_{2}<4$ for any $4 \leq a \leq 100$. The remaining small values of $v$ can be checked for solutions, using (6.1) and (6.2).

The only nontrivial solution we found was $a=4, a_{1}=4, a_{2}=-1, a_{3}=0$, which corresponds to the solution stated in the theorem.

For $a=3$ one can solve (2) similarly, starting from the equation

$$
(\alpha-1)^{a_{1}} \alpha^{a_{2}} \varepsilon_{0}^{a_{3}}=x-\alpha y
$$

instead of (4.2). Here, $\varepsilon_{0}=(\alpha-1 / \alpha)^{1 / 2}$, which together with $\alpha-1$ and $\alpha$ form a system of fundamental units in $\mathrm{Z}[\alpha]$ by Theorem 3.1.

The computation took a few minutes on an IBM PC computer. 
Remark. Solving a quartic Thue equation of unit rank 3, one usually has to reduce the upper bounds for the coefficients of four linear forms each involving four logarithms of algebraic numbers (see Pethő and Schulenberg [7], Steiner [9], and de Weger [14]). For this reason, the initial upper bound is considerably larger than the one we obtained.

Proof of Theorem 3. The proof can be performed as that of Theorems 1 and 2. The details are left to the reader.

Proof of Theorem 4. Let $n \in \mathbf{Z}$ be such that $(u, v)=\left(R_{n}, R_{n+1}\right)$ or $\left(R_{n+1}, R_{n}\right)$ is a solution of (1.5). Then $(u, v)=1$. We may assume $\eta>1$ without loss of generality. If $n<0$, then $R_{n}=R_{-n}$ if $\eta \eta^{\prime}=1$ and $R_{n}=(-1)^{n} R_{-n}$ if $\eta \eta^{\prime}=-1$, hence we may assume $n \geq 0$.

There exist $x, y \in \mathbf{Z}$ with $(x, y)=1, x \not \equiv y(\bmod 2)$ such that

$$
(u, v)= \begin{cases}\left(x y, x^{2}-y^{2}\right) & \text { if } u \text { is even, } \\ \left(x^{2}-y^{2}, 4 x y\right) & \text { if } u \text { is odd, }\end{cases}
$$

because (1.5) is a Pythagorean equation. Hence, if $u$ is even, then

$$
\begin{gathered}
x y=\frac{\eta^{n}-\eta^{\prime n}}{\eta-\eta^{\prime}} \text { or } \frac{\eta^{n+1}-\eta^{\prime n+1}}{\eta-\eta^{\prime}}, \\
x^{2}-y^{2}=\frac{\eta^{n+1}-\eta^{\prime n+1}}{\eta-\eta^{\prime}} \text { or } \frac{\eta^{n}-\eta^{\prime n}}{\eta-\eta^{\prime}} .
\end{gathered}
$$

Multiplying the first equation (7.1) by $\eta$ and subtracting it from the first equation (7.2), we get

$$
x^{2}-\eta x y-y^{2}=\eta^{\prime n} .
$$

Taking conjugates and multiplying the two resulting equations yields

$$
x^{4}-a x^{3} y-\left(2-\eta \eta^{\prime}\right) x^{2} y^{2}+a x y^{3}+y^{4}=\left(\eta \eta^{\prime}\right)^{n}= \pm 1,
$$

where $a=\left|\eta+\eta^{\prime}\right|$. The polynomial on the left-hand side of the last equation is exactly $f_{a}(x, y)$ if $\eta \eta^{\prime}=1$ and $g_{a}(x, y)$ if $\eta \eta^{\prime}=-1$.

Multiplying the second equation (7.2) by $\eta$, subtracting it from the second equation (7.1) and performing the same procedure as above, we get the same polynomial.

Similarly, for $u$ odd, $(x, y) \in \mathbf{Z}^{2}$ must be a solution of the equation

$$
h_{\eta \eta^{\prime}, a}(x, y)=x^{4}-4 a x^{3} y-\left(2-16 \eta \eta^{\prime}\right) x^{2} y^{2}+4 a x y^{3}+y^{4}= \pm 1 \text {. }
$$

It is easy to see that $h_{1, a}(x, y)=f_{a}(y-x, y+x)$ and $h_{-1, a}(x, y)=$ $-g_{a}(x-y, x+y)$.

Hence, Theorems 1, 2, and 3 imply the assertion of Theorem 4. 


\section{ACKNOWLEDGMENTS}

I thank I. Gaál for the modification of his and Schulte's program. I am grateful to M. Pohst for calling my attention to the method which enabled me to prove the results in $\S 3$. I also thank the referee for the several comments and improvements on an earlier draft of the manuscript.

\section{BIBLIOGRAPHY}

1. A. Baker and H. Davenport, The equations $3 x^{2}-2=y^{2}$ and $8 x^{2}-7=z^{2}$, Quart. J. Math. Oxford 20 (1969), 129-137.

2. J. Blass, A. M. W. Glass, D. Manski, D. B. Meronk, and R. P. Steiner, Constants for lower bounds for linear forms in the logarithms of algebraic numbers. II: The rational case, Acta Arith. 55 (1990), 15-22.

3. I. Gaál and N. Schulte, Computing all power integral bases of cubic fields, Math. Comp. $\mathbf{5 3}$ (1989), 689-696.

4. W. Ljunggren, On the representation of integers by binary biquadratic forms of a special class, Norsk Mat. Tidsskr. 26 (1944), 51-59.

5. T. Nagell, Sur les représentations de l'unité par les formes binaires biquadratiques du premier rang, Ark. Mat. 5 (1965), 477-521.

6. _ _ Sur les unités dans les corps biquadratiques primitifs du premier rank, Ark. Mat. 7 (1968), 359-394.

7. A. Pethö and R. Schulenberg, Effektives Lösen von Thue Gleichungen, Publ. Math. Debrecen 34 (1987), 189-196.

8. M. Pohst and H. Zassenhaus, Algorithmic algebraic number theory, Cambridge Univ. Press, 1989.

9. R. P. Steiner, On Mordell's equation $y^{2}-k=x^{3}$ : A problem of Stolarsky, Math. Comp. 46 (1986), 703-714.

10. R. J. Stroeker, On quartic Thue equations with trivial solutions, Math. Comp. 52 (1989), 175-187.

11. R. J. Stroeker and N. Tzanakis, On the application of Skolem's p-adic method to the solution of Thue equations, J. Number Theory 29 (1988), 166-195.

12. E. Thomas, Complete solutions to a family of cubic Diophantine equations, J. Number Theory 34 (1990), 235-250.

13. _ Solutions to families of cubic Thue equations. I, Preprint MSRI 07108-89.

14. B. M. M. de Weger, Algorithms for Diophantine equations, CWI Tract 65, 1989.

Mathematical Institute, Kossuth Lajos University, P. O. Box 12, 4010 Debrecen, HUNGARY 